\title{
Response bias reflects individual differences in sensory encoding
}

Dobromir Rahnev

School of Psychology, Georgia Institute of Technology, Atlanta, GA

Keywords: bias, criterion, perceptual decision making, sensory encoding, confidence, individual differences

\section{Author contributions}

D.R conceptualized of the study idea, analyzed the data, and wrote the manuscript.

\section{Acknowledgements}

This work was supported by the National Institute of Mental Health of the National Institutes of Health under Award Number R01MH119189 to D.R.

\section{Correspondence}

Dobromir Rahnev

Georgia Institute of Technology

654 Cherry Str. NW

Atlanta, GA 30332

E-mail: rahnev@psych.gatech.edu 


\begin{abstract}
Humans exhibit substantial biases in their decision making even in simple 2-choice tasks but the origin of these biases remains unclear. We hypothesized that one source of bias could be individual differences in sensory encoding. Specifically, if one stimulus category gives rise to an internal evidence distribution with higher variability, then responses should optimally be biased against that stimulus category. Therefore, response bias may reflect a previously unappreciated subjectto-subject difference in the shape of the internal evidence distributions. We tested this possibility by analyzing data from three different 2 -choice tasks $(\mathrm{N}=443,443$, 498). We found that, for all three tasks, response bias moved in the direction of the optimal criterion determined by each subject's idiosyncratic internal evidence variability. These results demonstrate that seemingly random variations in response bias can be driven by individual differences in sensory encoding and are thus partly explained by normative strategies.
\end{abstract}




\section{Statement of Relevance}

What is the origin of our decision biases? Centuries of research have shown that humans form preferences for one stimulus category over another in tasks that range from choosing a vacation destination to simple perceptual judgments. Such biases are typically seen as deficiencies to be avoided. However, here we examined whether response bias can reflect normative computations. Using perceptual decision making as a model system, we tested if decision bias reflects individual differences in sensory encoding. We analyzed the data from three previously published tasks ( $\mathrm{N}=443,443$, and 498) and applied mathematical modeling to determine the idiosyncratic way in which two stimulus categories are encoded by each subject. Our results showed that the response bias reflects the idiosyncratic sensory encoding in each individual, demonstrating that our decision biases are not necessarily deficiencies to be avoided but can normatively reflect how information is internally represented. 


\section{Introduction}

Humans have the propensity to prefer one stimulus category over another even in the context of simple perceptual tasks. Recent research has revealed that such perceptual biases have a substantial sensory component. For example, Linares, Aguilar-Lleyda, \& López-Moliner (2019) showed that response bias in a 2-choice task is driven by both sensory and decisional factors, with the sensory factor surprisingly being larger in magnitude. Other studies have further demonstrated that response bias depends on neuronal excitability (Iemi \& Busch, 2018), can be manipulated by optogenetic manipulation of V1 (Jin \& Glickfeld, 2019) and MT (Fetsch et al., 2018), and, in the case of continuous variables, can be predicted from the empirical form of the stimulus sensitivity (Wei \& Stocker, 2017). Together, this line of research demonstrates that response bias in perceptual experiments has a substantial sensory component.

However, previous studies provide limited insight into perhaps the most confounding aspect of response bias: the fact that different people can exhibit opposite biases for the exact same task. For example, people have been shown to have idiosyncratic perceptual biases that change with the spatial location of a stimulus on a display (Afraz, Pashkam, \& Cavanagh, 2010; Finlayson, Papageorgiou, \& Schwarzkopf, 2017; Kosovicheva \& Whitney, 2017; Moutsiana et al., 2016; Wexler, Duyck, \& Mamassian, 2015). Critically, these biases are stable over time but vary greatly from person to person. Similar findings have been reported with simpler tasks such as the existence of stable but idiosyncratic biases for presentation order 
of stimuli in 2-interval forced-choice tasks (García-Pérez \& Alcalá-Quintana, 2011) and even in basic 2-choice tasks that require subjects to identify whether a single stimulus is tilted clockwise or counterclockwise from vertical (Rahnev \& Denison, 2018; Rahnev, Nee, Riddle, Larson, \& D’Esposito, 2016).

What causes such persistent and idiosyncratic biases in simple perceptual tasks? We previously proposed that individual differences in stimulus encoding may partly explain the response biases in 2-choice perceptual experiments (Rahnev \& Denison, 2018). In such experiments, bias is typically assessed using signal detection theory, which can separate sensitivity (quantified by the measure of sensitivity, $d^{\prime}$ ) from the response bias (quantified by the measure of criterion, $c$ ) (Green \& Swets, 1966). In the absence of uneven priors or payoffs, accuracy is maximized by setting the criterion to $c=0$, which corresponds to an unbiased response strategy. However, this approach assumes that the underlying distributions for the two stimulus categories have equal variance. If the equal variance assumption is violated, then the optimal criterion is no longer at $c=0$. Based on these considerations, we reasoned that individual variability in the variability of the internal distributions for each stimulus category may drive at least some of the idiosyncratic response bias observed in 2-choice tasks (Rahnev \& Denison, 2018).

What is the exact relationship between the optimal criterion and the variance of the internal distributions of evidence? Let the distributions of internal evidence for categories 1 and 2 have standard deviations of $\sigma_{1}$ and $\sigma_{2}$, respectively. Assume that 
$\sigma_{1}<\sigma_{2}$. In this scenario, the two distributions no longer intersect half-way between their means; instead, they intersect at a point that is closer to the mean for category 2 (Figure 1). (Note that there is also a second point of intersection that does not lie in-between the two distributions' means. This point should be taken into account by the ideal observer but ignoring it typically has only a minor effect on subjects' responses. We return to this issue in the Discussion.) If the difference in variability is ignored and equal variance is assumed during data analysis, then the optimal strategy, which is to place the criterion at the first point of intersection, would appear biased. Specifically, the optimal criterion, $c_{o p t}$, would be greater than zero (Figure 1). On the other hand, when $\sigma_{1}>\sigma_{2}$, the same logic dictates that $c_{\text {opt }}$ will be smaller than 0. Failing to consider the possibility of internal distributions of unequal variance would thus result in categorizing the otherwise optimal criterion, $c_{o p t}$, as "biased" because it will deviate from the value of 0 that is optimal under the assumption of equal variance.
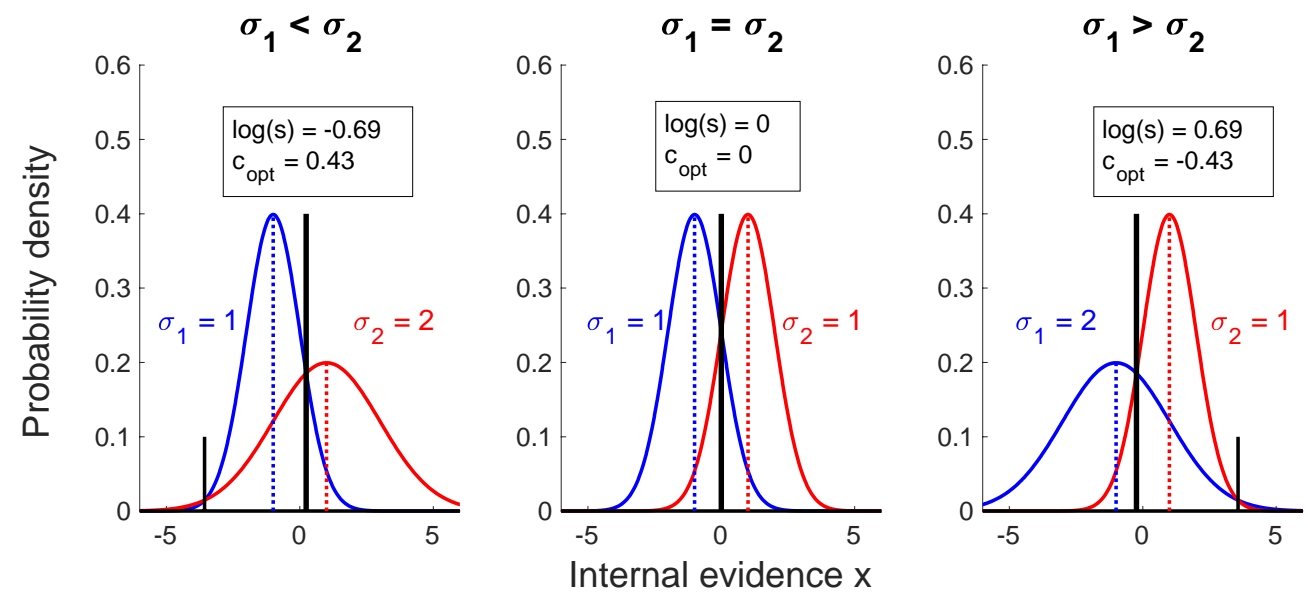

Figure 1. The dependence of the optimal criterion on sensory encoding. The three panels depict internal Gaussian distributions of evidence for categories 1 and 2 with 
standard deviations $\sigma_{1}$ and $\sigma_{2}$, respectively. The ratio of the two standard deviations, $s=\frac{\sigma_{1}}{\sigma_{2}}$, varies from .5 in the left panel to 1 in the middle panel, to 2 in the right panel. The logarithm of $s$, therefore, changes from -.69 to 0 to .69 . The corresponding optimal criterion, $c_{o p t}$, (long solid vertical line; see Methods for details on its computation) changes from .43 to 0 to -.43. The reason for the opposite relationship between $\log (s)$ and $c_{\text {opt }}$ is that a distribution with a higher standard deviation (e.g., $\sigma_{2}$ in the left panel) brings the point of intersection between the two Gaussian distributions towards its own mean. Taken together, the three panels demonstrate that the negative relationship between $\log (s)$ and $c_{\text {opt }}$. Note the existence of a second point of intersection in the left and right panels (short vertical line). An optimal observer would set a second criterion at the second intersection point and flip the responses beyond that criterion. However, it is unclear whether human observers do this (Macmillan \& Creelman, 2005). Moreover, such second criterion would typically make little practical difference. Indeed, in the left and right panels above, only $0.81 \%$ of the distributions lie beyond the second intersection point, which, in turn, is a factor of 9 larger than what we observed in the empirical data. Therefore, we ignore the influence of this second point of intersection but return to this issue in the Discussion.

How can we test whether the response bias of individual subjects indeed reflects their idiosyncratic sensory encoding as measured by the variance of the internal distributions of evidence? As Figure 1 demonstrates, we can expect a simple relationship between the ratio of the two standard deviations, $s=\frac{\sigma_{1}}{\sigma_{2}}$ (which can be computed in a straightforward manner for experiments that collect confidence ratings, see Macmillan \& Creelman, 2005), and the optimal criterion $c_{o p t}$ : as $s$ increases, $c_{o p t}$ should decrease. Therefore, whether the actual criterion $c$ reflects the sensory encoding specified by the parameter $s$ can be assessed by conducting an across-subject correlation between $\log (s)$ and $c$. (Note that because the parameter $s$ is a ratio and thus not normally distributed, it is more appropriate to use its natural $\operatorname{logarithm}, \log (s)$, for statistical tests.) 
Based on power calculations, which suggested the need for experiments with at least 250 subjects and 150 trials per subject (see Methods), we selected three tasks from the recently published Confidence Database (Rahnev et al., 2020) for analysis. All three tasks had $\mathrm{N}>400$ and included simple 2-choice perceptual judgments thus making them ideal to test the current hypothesis. To anticipate, we confirmed the presence of stable idiosyncratic biases and found that $\log (s)$ and $c$ were negatively correlated in all three tasks, suggesting that response bias indeed reflects individual differences in sensory encoding. 


\section{Methods}

\section{Dataset selection}

We searched for datasets in which subjects performed 2-choice perceptual tasks with confidence ratings. Critically, based on power analyses (see Power analyses section), we required the datasets to have at least 250 subjects each completing at least 150 trials per task. We first examined the 149 datasets included in the recently published Confidence Database (Rahnev et al., 2020) as of July 2020. Two datasets met the above criteria: "Haddara_2020" and "Rouault_2018_Expt1" (the names of these datasets are as they appear in the Confidence Database). We additionally conducted an extensive literature search for additional datasets but could not find any others that met the above criteria.

Therefore, we performed all analyses on the tasks from these two datasets. The first dataset, originally reported in Haddara \& Rahnev (2020), consists of two separate tasks, considered here as Task 1 and Task 2 . The second dataset, originally reported as Experiment 1 in Rouault, Seow, Gillan, \& Fleming (2018), consists of a single task, considered here as Task 3. The Haddara \& Rahnev dataset has 443 subjects all of which were included in the Confidence Database regardless of data quality. On the other hand, the Rouault et al. dataset originally had 663 subjects but the authors removed 165 subjects (24.9\%) based on exclusion criteria involving task comprehension and performance leaving them with 498 subjects. The data from these 498 subjects were posted on the Confidence Database and therefore here we 
only analyze these data. All subjects provided informed consent and the experiments were approved by the local Institutional Review Boards.

\section{Experimental designs}

Complete details about Tasks 1 and 2 are available in the original paper (Haddara \& Rahnev, 2020). Briefly, subjects indicated whether the letter X or O (Task 1) or the color red or blue (Task 2) occurred more frequently in a $7 \times 7$ grid. In both tasks, each trial began with a fixation period (500 ms), followed by stimulus presentation (500 ms), untimed perceptual judgment, and untimed confidence rating provided on a 4-point scale (Figure 2a,b). The two tasks were adapted from Rahnev, Koizumi, McCurdy, D'Esposito, \& Lau (2015). In Task 1, approximately half of the subjects received trial-by-trial feedback, while the other half received no such feedback. The feedback screen consisted of the word "Correct" or "Wrong" and was presented for $500 \mathrm{~ms}$. The group that did not receive trial-by-trial feedback saw a fixation cross for 500 ms instead of the feedback screen. All subjects were analyzed together regardless of whether they received feedback. No subject received feedback in Task 2. The more frequent stimulus within the $7 \times 7$ grid was presented in 31 locations in Task 1 and 27 locations in Task 2. Task 1 consisted of 330 trials per subject, whereas Task 2 consisted of 150 trials per subject. Both tasks were organized in blocks of 30 trials and subjects could take a break at the end of each block. 
a

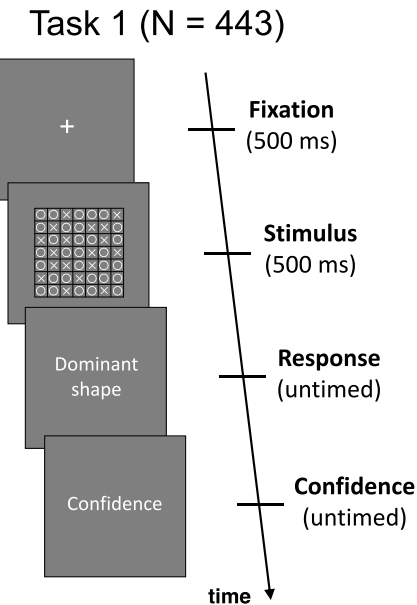

b $\quad$ Task $2(\mathrm{~N}=443)$

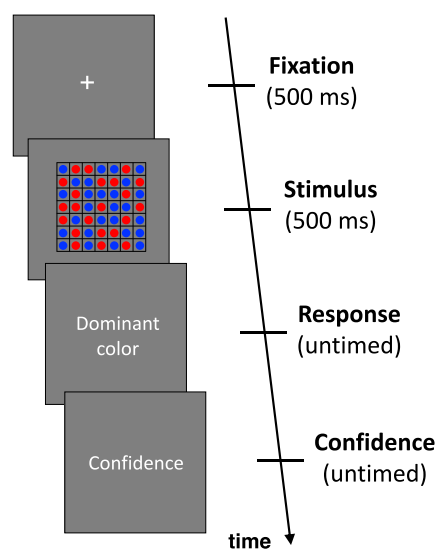

C $\quad$ Task $3(\mathrm{~N}=498)$

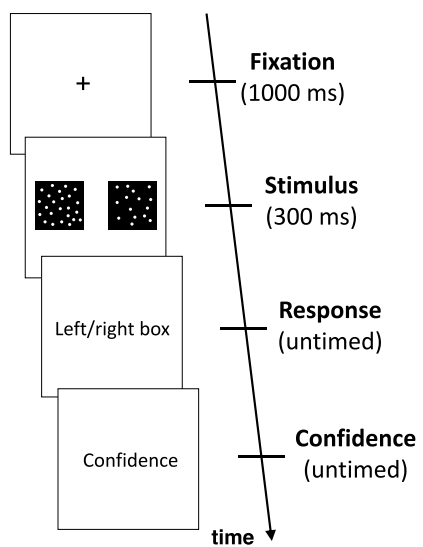

Figure 2. Experimental tasks. Each task began with a fixation cross, after which a stimulus was presented for a brief period (either 500 or $300 \mathrm{~ms}$ ), and finally subjects indicated their perceptual judgment and confidence rating with separate, untimed button presses. Subjects judged (a) whether the letter X or 0 was more frequent (Task 1), (b) whether the color red or blue was more frequent (Task 2), or (c) whether the left or right box contained more white dots (Task 3). Confidence was given on a 4-point scale in Tasks 1 and 2, and on an 11-point scale in Task 3.

Complete details about Task 3 are available in the original paper (Rouault et al., 2018). Briefly, subjects indicated which of two simultaneously presented black boxes had the highest number of white dots. Each trial began with a fixation period (1,000 ms), followed by stimulus presentation (300 ms), untimed perceptual judgment, and untimed confidence rating provided on an 11-point scale (Figure 2c). No feedback was provided during the task. One box was always half-filled (313 dots out of 625 positions), while the other box contained an increment of +1 to +70 dots compared to the standard. Task 3 consisted of 210 trials per subject organized in 5 blocks. 
All data were collected online using Amazon Mechanical Turk. The experiments were performed using jsPsych (version 5.0.3 for Tasks 1 and 2, version 4.3 for Task $3)$.

\section{$\underline{\text { Subject selection }}$}

The analyses here necessitated the estimation of the parameters of a signal detection theoretic model with unequal variance. However, the parameters of this model are difficult to estimate in the presence of extreme biases or if performance is too low or too high. Therefore, we excluded subjects who gave the same response or the same confidence rating on more than $95 \%$ of all trials or had accuracy lower than $55 \%$ or higher than $95 \%$ correct. In addition, we also excluded individual trials with response times that were faster than $200 \mathrm{~ms}$ or slower than 2 seconds. These criteria were identical to the exclusion criteria used in Haddara \& Rahnev (2020).

The selection criteria resulted in the exclusion of 68 subjects in Task 1 (15.3\%), 79 subjects in Task 2 (17.8\%), and 5 subjects in Task $3(1 \%)$. We note that the much lower exclusion rate in Task 3 is due to the fact that the original authors already excluded a number of subjects using somewhat overlapping exclusion criteria. The exclusions in Tasks 1 and 2 were made independently for each task.

\section{$\underline{\text { Analyses }}$}

We were interested in whether subjects can take into account the idiosyncratic shapes of their internal distributions when making perceptual decisions. As 
explained in the Introduction, this would be reflected in a correlation between the criterion, $c$, and the logarithm of the SD ratio of the internal distributions, $\log (s)$. We note that Haddara and Rahnev (2020) also examined response criterion using the same data but focused on how trial-by-trial feedback affects bias. They found that bias was reduced in the group that received feedback but did not examine the source of the idiosyncratic differences in this bias.

To address whether individual differences in sensory encoding affect response bias, we first computed the parameters $d^{\prime}$ and $c$ which assume that the Gaussian distributions of evidence for the two stimulus categories have equal variance. To do so, we calculated the hit rate (HR) and false alarm rate (FAR) by treating the letter $\mathrm{X}$ in Task 1 , the blue color in Task 2 , and the right box in Task 3 as the targets. Then, $d^{\prime}$ and $c$ were calculated using the following formulas:

$d^{\prime}=\Phi^{-1}(H R)-\Phi^{-1}(F A R)$

and

$c=-\frac{1}{2}\left(\Phi^{-1}(H R)+\Phi^{-1}(F A R)\right)$

where $\Phi^{-1}$ is the inverse of the cumulative standard normal distribution that transforms HR and FAR into z-scores. In cases where HR or FAR were equal to 0 or 1 , a standard correction was applied such that, if estimated from $k$ trials, values of 0 
were replaced with $\frac{1}{2 k}$, whereas values of 1 were replaced with $1-\frac{1}{2 k}$ (Macmillan \& Creelman, 2005). Note that negative $c$ values indicate a bias for the letter X (Task 1), the color blue (Task 2), and the right box (Task 3), whereas positive $c$ values indicate a bias for the letter 0 (Task 1), the color red (Task 2), and the left box (Task $3)$.

The computations above assume that both stimulus categories produce internal evidence distributions with equal variance. However, this assumption does not hold in general. For example, the target distribution has a higher variance in both detection and memory tasks (Macmillan \& Creelman, 2005; Rahnev et al., 2011). More generally, even when neither stimulus category results in higher variability across the whole group, it is likely that individual subjects exhibit higher variability for one or the other stimulus category with the category changing from subject to subject.

To compute the relative variance of the distributions for each stimulus category, we used standard techniques based on subjects' confidence ratings (Green \& Swets, 1966). For each confidence and decision criterion, we computed the HR and FAR. We then computed zHR and zFAR using the formulas $z H R=\Phi^{-1}(H R)$ and $z F A R=\Phi^{-1}(F A R)$, and found the line of best fit for the plot of zHR against zFAR values. As has been demonstrated previously (Macmillan \& Creelman, 2005), the slope, $s$, of this line is equal to the ratio of the standard deviations of the internal distributions for categories 1 and 2: $s=\frac{\sigma_{1}}{\sigma_{2}}$. Because only the ratio of the two 
standard deviations is fixed and their actual values do not matter, without loss of generality, we can set $\sigma_{1}=s$ and $\sigma_{2}=1$. Given these standard deviations, we can also obtain the distance between the means of the two Gaussian distributions as the intercept of the line of best fit above. Because only the distance between the two means matters, without loss of generality, we can set the mean of the Gaussian distribution for category 1 to 0 (i.e., $\mu_{1}=0$ ), and therefore the mean of the Gaussian distribution for category 2 becomes the intercept of the line of best fit, which we denote as $\mu$ (i.e., $\mu_{2}=\mu$ ).

Having determined the means and standard deviations for the Gaussian distributions for each stimulus category, we can now compute the location of the optimal decision criterion, $x_{\text {opt }}$. This is the location where the two Gaussian distributions intersect, and therefore their probability density functions are equal to each other:

$\frac{1}{\sqrt{2 \pi \sigma_{1}^{2}}} e^{-\frac{1}{2}\left(\frac{x-\mu_{1}}{\sigma_{1}}\right)^{2}}=\frac{1}{\sqrt{2 \pi \sigma_{2}^{2}}} e^{-\frac{1}{2}\left(\frac{x-\mu_{2}}{\sigma_{2}}\right)^{2}}$

To compute $x_{o p t}$, we can solve for $x$ :

$$
\frac{\sqrt{2 \pi \sigma_{2}^{2}}}{\sqrt{2 \pi \sigma_{1}^{2}}}=e^{\frac{1}{2}\left(\frac{x-\mu_{1}}{\sigma_{1}}\right)^{2}-\frac{1}{2}\left(\frac{x-\mu_{2}}{\sigma_{2}}\right)^{2}}
$$




$$
\begin{gathered}
\log \left(\frac{\sigma_{2}}{\sigma_{1}}\right)=\frac{\left(x-\mu_{1}\right)^{2} \sigma_{2}^{2}-\left(x-\mu_{2}\right)^{2} \sigma_{1}^{2}}{2 \sigma_{1}^{2} \sigma_{2}^{2}} \\
2 \sigma_{1}^{2} \sigma_{2}^{2} \log \left(\frac{\sigma_{2}}{\sigma_{1}}\right)=x^{2}\left(\sigma_{2}^{2}-\sigma_{1}^{2}\right)+2 x\left(\sigma_{1}^{2} \mu_{2}-\sigma_{2}^{2} \mu_{1}\right)+\left(\mu_{1}^{2} \sigma_{2}^{2}-\mu_{2}^{2} \sigma_{1}^{2}\right) \\
x^{2}\left(\sigma_{2}^{2}-\sigma_{1}^{2}\right)+2 x\left(\sigma_{1}^{2} \mu_{2}-\sigma_{2}^{2} \mu_{1}\right)+\left(\mu_{1}^{2} \sigma_{2}^{2}-\mu_{2}^{2} \sigma_{1}^{2}-2 \sigma_{1}^{2} \sigma_{2}^{2} \log \left(\frac{\sigma_{2}}{\sigma_{1}}\right)\right)=0
\end{gathered}
$$

When $\sigma_{1}=\sigma_{2}$, we obtain $x=\frac{\mu_{1}+\mu_{2}}{2}$. This corresponds to the familiar case of equal variance where the optimal criterion is located halfway between the peaks of the two distributions. However, when $\sigma_{1} \neq \sigma_{2}$, the equation above has two solutions corresponding to the two locations where the two Gaussian distributions intercept:

$x_{1,2}=\frac{\left(\sigma_{2}^{2} \mu_{1}-\sigma_{1}^{2} \mu_{2}\right) \pm \sqrt{\left(\sigma_{1}^{2} \mu_{2}-\sigma_{2}^{2} \mu_{1}\right)^{2}-\left(\sigma_{2}^{2}-\sigma_{1}^{2}\right)\left(\mu_{1}^{2} \sigma_{2}^{2}-\mu_{2}^{2} \sigma_{1}^{2}-2 \sigma_{1}^{2} \sigma_{2}^{2} \log \left(\frac{\sigma_{2}}{\sigma_{1}}\right)\right)}}{\sigma_{2}^{2}-\sigma_{1}^{2}}$

Note that if we consider the case where $\sigma_{1}=s, \sigma_{2}=1, \mu_{1}=0$ and $\mu_{2}=\mu$, equation 4 simplifies to

$x_{1,2}=\frac{-\mu s^{2} \pm \sqrt{\mu^{2} s^{4}+\left(1-s^{2}\right)\left(\mu^{2} s^{2}-2 s^{2} \log (s)\right)}}{1-s^{2}}$

Note that the solution $x_{1}$ (where the two expressions in the nominator are added) lies between the two means, whereas the solution $x_{2}$ (where the two expressions 
are subtracted) is typically an outlier and lies far from both means: it is a large negative value when $s<1$ and a large positive value when $s>1$. The optimal decision strategy is to place criteria at both $x_{1}$ and $x_{2}$ and then choose one stimulus category for values between $x_{1}$ and $x_{2}$, and the other category for values outside of this range. However, across all subjects in the three tasks examined here, only $0.09 \%$ (that is, the extreme tail) of the area of the internal distributions lied beyond $x_{2}$. Thus, an ideal observer (Knoblauch \& Maloney, 2012) would respond differently on average once every 1,097 trials compared to an observer who simply ignores $x_{2}$. Given that it is theoretically questionable whether human observers can implement the decision strategy prescribed by the optimal observer (Macmillan \& Creelman, 2005 ) and that the ideal observer would anyway produce a very similar pattern of responses, we implemented an observer model that only places a criterion at the location of the first solution, $x_{1}$. Therefore, we refer to $x_{1}$ as the location of the optimal criterion and call it $x_{\text {opt }}$.

We can then compute the value, $c_{\text {opt }}$, that the optimal criterion would have in standard analyses that assume equal variance for the two Gaussian distributions:

$c_{o p t}=-\frac{1}{2}\left(\frac{\mu_{2}-x_{o p t}}{\sigma_{2}}+\frac{\mu_{1}-x_{o p t}}{\sigma_{1}}\right)$

Specifically, $c_{\text {opt }}$ is the value of the optimal criterion if bias is to be computed in the standard way using equation 2 . 
Having obtained the ratio of the standard deviations for the two categories, $s$, and the value of the optimal criterion, $c_{o p t}$, we could then explore how these two quantities relate to the actual location of the decision criterion, $c$. Specifically, if subjects have access to the fact that the two stimulus categories result in internal distributions of different variances, then the criterion $c$ that they use should correlate negatively with the ratio $s$ but positively with the optimal criterion $c_{\text {opt }}$. To test if this is indeed the case, for each of the three tasks, we correlated $c$ with both $\log (s)$ and $c_{\text {opt }}$. We used $\log (s)$ because $s$ is on a $\log$ scale such that $s=y$ and $s=\frac{1}{y}$ correspond to equivalent scenarios (where the category labels are simply switched), which is correctly captured when taking its $\operatorname{logarithm}($ since $\log (y)=$ $\left.-\log \left(\frac{1}{y}\right)\right)$. In addition, the fact that $s$ is a ratio means that its distribution has a heavy skew to the right, whereas the distribution of $\log (s)$ is symmetric and approximately Gaussian. Finally, we excluded values of $s$ smaller than $\frac{1}{3}$ and larger than 3 as outliers. These exclusions resulted in removing 3 subjects from Task 1 (0.8\%), 16 subjects from Task 2 (4.4\%), and 5 subjects from Task 3 (1\%).

We note that while our analyses are conducted within the framework of signal detection theory, we suspect that similar results would be obtained if the analyses were conducted within different frameworks such as, for example, sequential sampling (Forstmann, Ratcliff, \& Wagenmakers, 2016) or exemplar-storage models (McKinley \& Nosofsky, 1995). Therefore, the critical idea here is not the use of the 
specific signal detection metrics but rather the relating of idiosyncratic biases to individual differences in sensory encoding.

\section{Power analyses}

Demonstrating a significant negative correlation between $\log (s)$ and $c$ faces at least two challenges. First, both $\log (s)$ and $c$ require a large number of trials per subject for accurate estimation. Second, most subjects exhibit values of $\log (s)$ and $c$ close to zero, which makes it hard to establish how these two quantities correlate with each other (the limited ranges make it harder to detect a significant correlation). To gain insight into the severity of these challenges, we performed simulations to quantify the power of different experimental setups to reveal a significant correlation between $\log (s)$ and $c$ for cases when the criterion $c$ closely reflects the optimal criterion $c_{\text {opt }}$. We ran 64 sets of simulations by varying both the number of subjects and the number of trials per subject from 50 to 400 in steps of 50 . For each set of simulations, we generated 1,000 individual experiments with the corresponding number of subjects and number of trials per subject.

For each subject of each simulated experiment, without loss of generality, we set $\mu_{1}=0, \mu_{2}=\mu, \sigma_{1}=s$, and $\sigma_{2}=1$. The values of $\mu$ were then sampled from a normal distribution with a mean of 1.5 and SD of .5, whereas the values of $s$ were sampled so that $\log (s)$ comes from a normal distribution with a mean of 0 and SD of .15. We chose these values based on the values empirically observed in the 3 tasks reported here. In addition, the location of the decision criterion was sampled from a normal 
distribution centered on the optimal criterion location for the subject and a standard deviation of.3. Finally, we determined the locations of three confidence criteria (so that confidence would be given on a 4-point scale) for each subject such that the location of each criterion was greater than the preceding criterion by a value sampled from a uniform distribution $U(0,1)$.

For each simulated experiment, we computed $c$ and $\log (s)$ based on the simulated data alone (without any reference to the true generating parameters). We then correlated these two values across subjects and reported the percent of simulated experiments (among the 1,000 for each simulation set) for which $\mathrm{r}<0$ and $\mathrm{p}<.05$ (Figure 3).

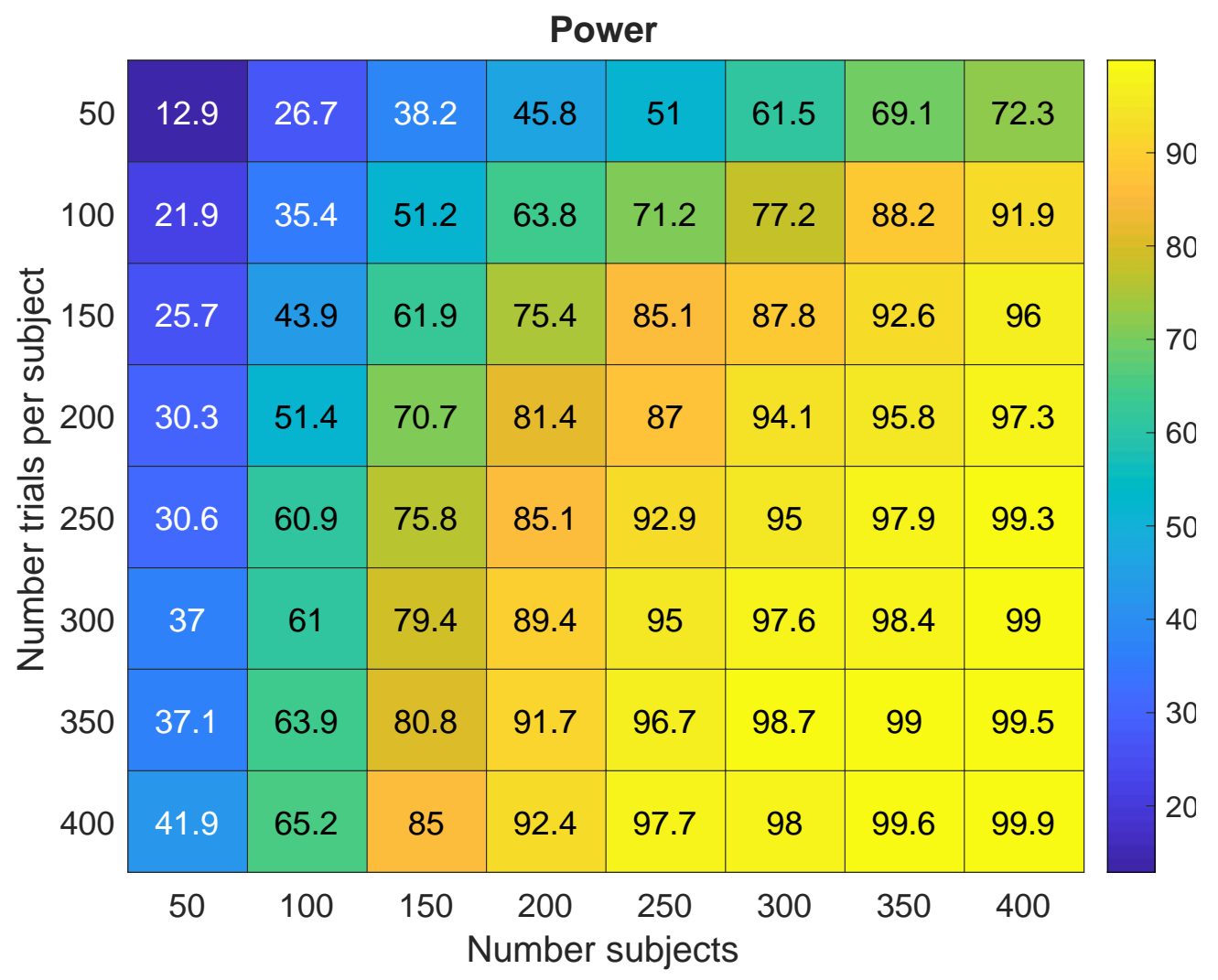


Figure 3. Power for observing a significantly negative correlation between $\log (s)$ and $c$ when the criterion $c$ reflects the optimal criterion $c_{\text {opt }}$. Each cell of the table represents the percent of expected significant correlations for a given combination of number of subjects and number of trials per subject. The values were obtained via simulations, which is why there are several inversions where a cell with a higher number of trials is shown to have lower power than a cell with a higher number of trials. Based on the estimated power, it appears that experiments need at least 250 subjects and at least 150 trials per subject to have sufficient power to uncover a significant negative correlation between $\log (s)$ and $c$.

The values in Figure 3 should monotonically increase within each row and column. However, since these values were produced using simulations, several inversions can be observed. Finally, we note that the obtained power estimates depend on the values of the parameters chosen for the simulations (mainly the variability of $\log (s)$ and the variability of the location of the actual criterion around the optimal criterion). Nevertheless, given that all of the values chosen here are based on our empirically observed data, the obtained power for each set of simulations is likely to be a reasonable approximation of the true power.

\section{Data and code availability}

All data and codes for the analyses have been made freely available at https://osf.io/r4gz3/(Rahnev, 2020). 


\section{Results}

We investigated whether response bias reflects individual differences in how different stimulus categories are encoded by each subject. We previously predicted that response bias may be partly driven by the fact that the ratio, $s$, of the standard deviations of the two internal distributions in 2-choice tasks likely varies between subjects (Rahnev \& Denison, 2018). Specifically, a larger standard deviation for a given category would optimally result in a response criterion that is shifted towards the mean of that distribution (Figure 1), thus resulting in a negative correlation between $\log (s)$ and the criterion $c$. We tested for this relationship using two different datasets (Haddara \& Rahnev, 2020; Rouault et al., 2018) made available as part of the Confidence Database (Rahnev et al., 2020) that consisted of three separate tasks ( $\mathrm{N}=443,443$, and 498).

We first examined whether there is evidence for the existence of stable idiosyncratic differences in response bias. To this end, we computed the criterion $c$ for odd and even trials within each subject, and correlated these values across subjects for each of the three tasks. We found that the two criterion values exhibited a very high degree of correlation $($ Task 1: $r=0.73, p=1.3 e-63,95 \% \mathrm{CI}=[0.68,0.77]$; Task 2: $\mathrm{r}=$ 0.73, $p=8.8 \mathrm{e}-61,95 \% \mathrm{CI}=[0.67,0.77]$; Task $3: \mathrm{r}=0.81, \mathrm{p}=4.7 \mathrm{e}-117,95 \% \mathrm{CI}=$ $[0.78,0.84]$; Figure 4, upper row). These results clearly demonstrate that response bias can be reliably calculated and that similar biases emerge when independent sets of trials are analyzed. We further examined whether these criterion values remain stable over the time course of each experiment by computing the correlation 
between the criterion $c$ derived separately for the first and second half of all trials in a given subject. We again found positive correlations for all three tasks (Task 1: $r=$ $0.5, p=2.1 \mathrm{e}-25,95 \% \mathrm{CI}=[0.42,0.57] ;$ Task $2: \mathrm{r}=0.56, \mathrm{p}=1.3 \mathrm{e}-31,95 \% \mathrm{CI}=[0.49$, 0.63]; Task 3: $\mathrm{r}=0.6, \mathrm{p}=4.9 \mathrm{e}-49,95 \% \mathrm{CI}=[0.54,0.65]$; Figure 4, lower row), though the strength of these correlations was predictably slightly lower compared to the correlations between odd and even trials. Overall, these results point to the existence of stable, idiosyncratic biases that vary substantially between different subjects.
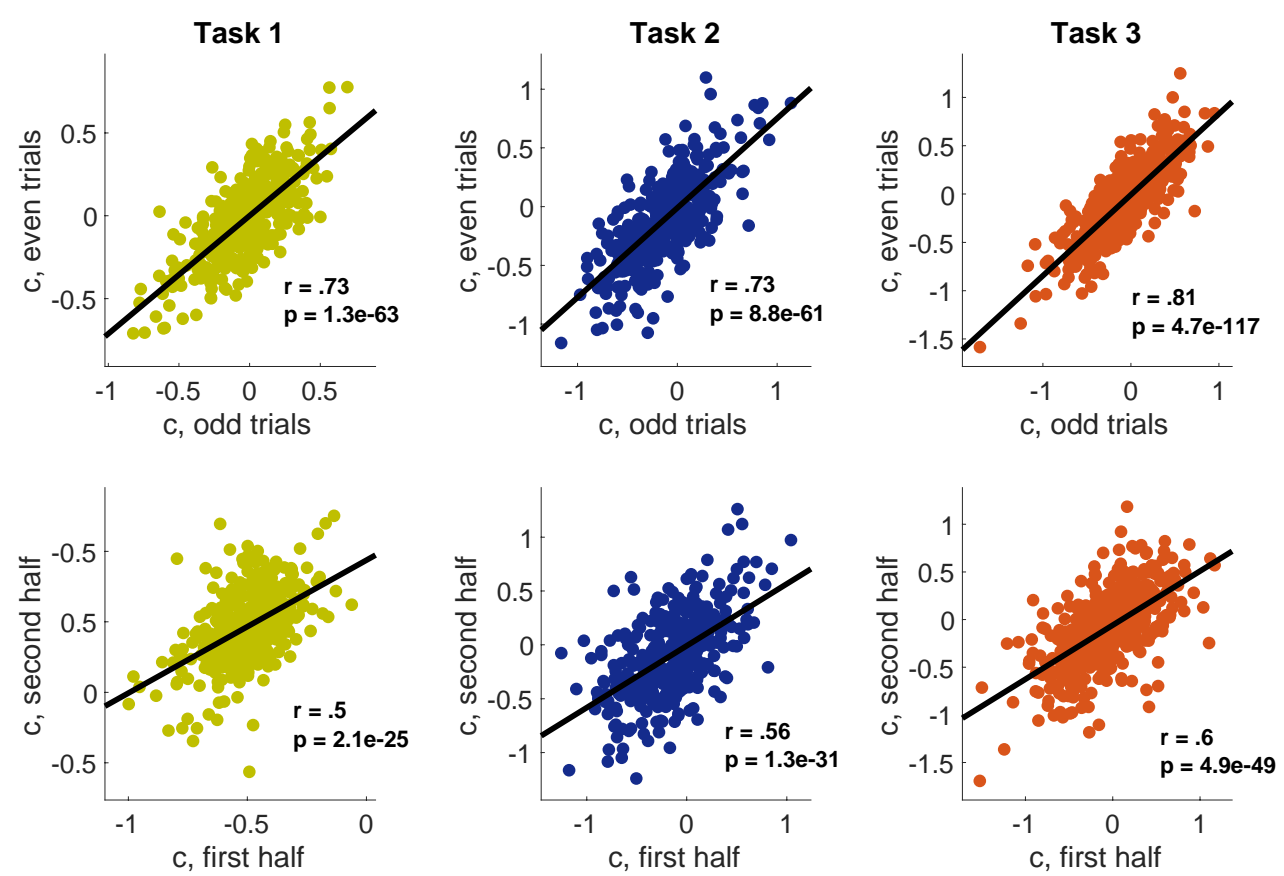

Figure 4. Stable idiosyncratic biases in simple 2-choice tasks. We observed significantly positive correlations between the criterion $c$ computed separately for odd and even trials (upper row), as well as for the first and second half of all trials in a given subject (lower row). The strong correlations were present for each of the three tasks and suggest that the individual bias for each subject is stable over time. 
Critically, we tested whether these stable, idiosyncratic response biases reflected individual differences in sensory encoding. As explained in Figure 1, subjects with higher (lower) values of $\log (s)$ would optimally have lower (higher) values of the criterion $c$. Therefore, if subjects are sensitive to their individual sensory encoding, then we should observe a negative across-subject correlation between $\log (s)$ and $c$. Consistent with this prediction, we found significantly negative correlations between $\log (s)$ and $c$ for each one of the three tasks (Task $1: \mathrm{r}=-0.16, \mathrm{p}=0.002$, $95 \% \mathrm{CI}=[-0.25,-0.06]$; Task 2: $\mathrm{r}=-0.14, \mathrm{p}=0.009,95 \% \mathrm{CI}=[-0.24,-0.04]$; Task 3: $\mathrm{r}$ $=-0.25, \mathrm{p}=2.9 \mathrm{e}-08,95 \% \mathrm{CI}=[-0.33,-0.16] ;$ Figure 5 , upper row), thus providing direct support for the notion that response bias reflects individual differences in sensory encoding.
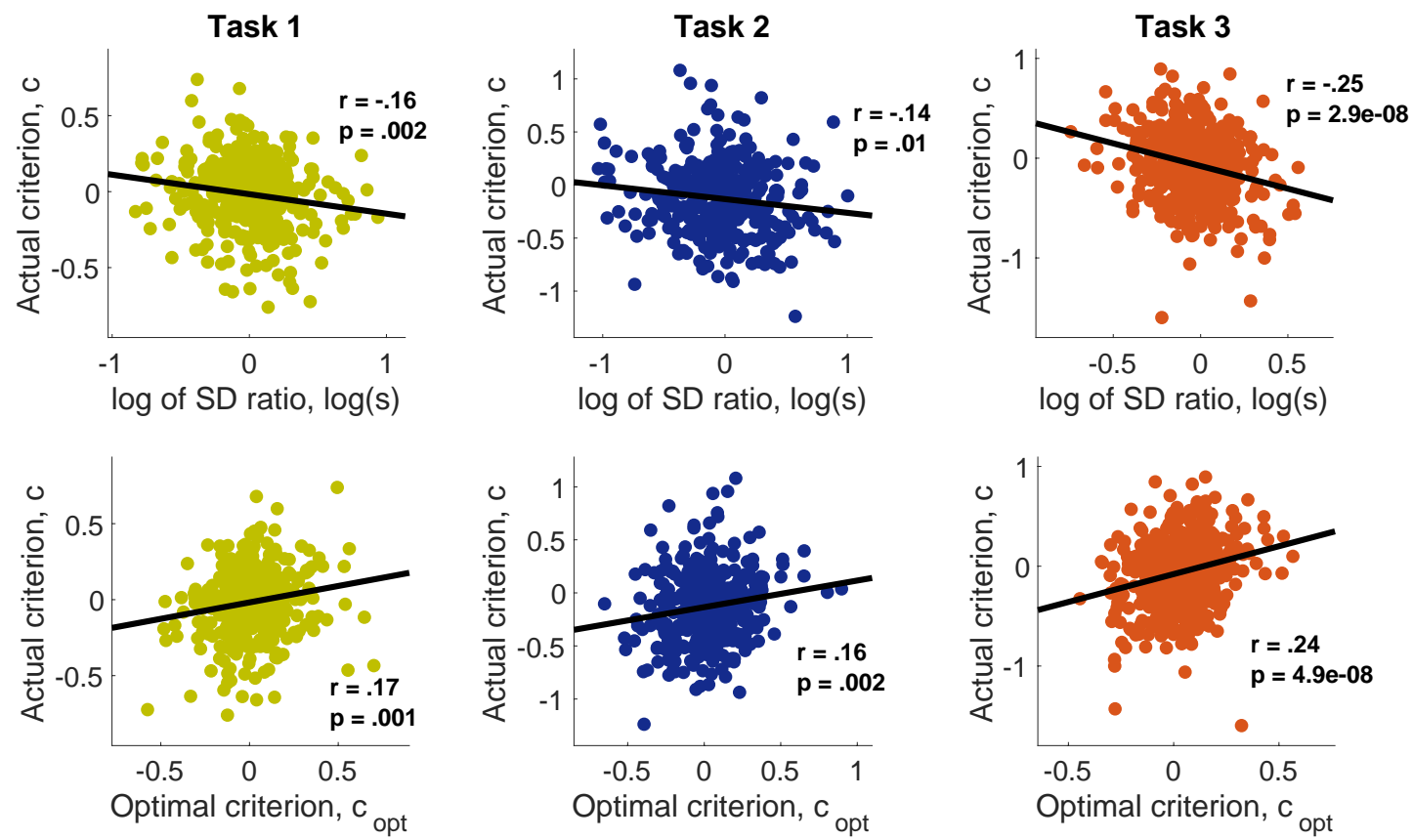

Figure 5. Response bias reflects individual differences in sensory encoding. We correlated criterion $c$ with both the measure of relative variability in the internal distributions, $\log (s)$, and the optimal criterion, $c_{\text {opt }}$. We observed significantly 
negative correlations between $\log (s)$ and $c$ for, as well as significantly positive correlations between $c_{o p t}$ and $c$. These results were observed for each of the three tasks, demonstrating that response bias reflects the subject-by-subject differences in sensory encoding.

It should be noted that the correlation between $\log (s)$ and $c$ does not consider the sensitivity of each observer. Therefore, to account for the varying sensitivity between our subjects, we directly estimated the optimal criterion $c_{o p t}$ for each subject based on both the ratio of the two standard deviations of the internal distributions, $s$, and the distance between their means, $\mu$. We then correlated the actual criterion, $c$, with the optimal criterion, $c_{o p t}$. We found significant positive correlations between these two quantities for each of the three tasks (Task 1: $r=$ $0.17, \mathrm{p}=0.001,95 \% \mathrm{CI}=[0.07,0.27] ;$ Task $2: \mathrm{r}=0.16, \mathrm{p}=0.002,95 \% \mathrm{CI}=[0.06$, 0.27]; Task 3: $\mathrm{r}=0.24, \mathrm{p}=4.9 \mathrm{e}-08,95 \% \mathrm{CI}=[0.16,0.33]$; Figure 5, lower row). Together, these results demonstrate that subjects can take the nature of their idiosyncratic sensory encoding into account when making perceptual decisions.

Finally, we performed several control analyses. First, we confirmed the existence of a negative relationship between $\log (s)$ and $c_{\text {opt }}$. Indeed, there was a strong negative correlation between these two variables $(\mathrm{r}<-.89, \mathrm{p}<\mathrm{e}-134$ for all three tasks). Second, we confirmed that the pattern of results in Figure 5 can be replicated in our simulations. Using the same methods and parameters from the power computations in Figure 3, we simulated an experiment with 400 subjects and 200 trials per subject, thus roughly matching our Tasks $1-3$. We then analyzed the simulated data 
in the same way as the actual experimental data and confirmed that the resulting scatterplots appeared very similar to the results in Figure 5 (see Supplementary Figure 1). These simulations confirm that the results we observed could plausibly be obtained if subjects' criterion indeed followed the optimal criterion placement. Finally, given the relatively small effect sizes obtained in Figure 5 (average $r$ values were -.18 for the correlations between $c$ and $\log (s)$, and.19 for the correlations between $c$ and $c_{o p t}$ ), we sought to examine what is a reasonable upper bound for these correlations in our experiments. We repeated the same simulations from the previous analysis 1,000 times and assessed the relationship between the estimated criterion $c$ with the true underlying parameters $s_{\text {true }}$ (used to generate the simulated data) and $c_{\text {true opt }}$ (computed directly based on the value of $s_{\text {true }}$ ). We found average correlations coefficients of $r=-.33(S D=.05)$ for the correlation between $c$ and $\log \left(s_{\text {true }}\right)$, and $\mathrm{r}=.36(\mathrm{SD}=.05)$ for the correlation between $c$ and $c_{\text {true opt }}$ (Supplementary Figure 2), suggesting that the maximum correlations that could be expected are only about twice as large as the ones actually observed. A major factor for this relatively low upper bound is the presence of estimation noise when computing SDT parameters from limited data. 


\section{Discussion}

It is well known that humans can appropriately place decision criteria, often in a close-to-optimal fashion (Ashby \& Maddox, 2005; McKinley \& Nosofsky, 1995). However, even when performing the same task, individual subjects tend to have stable, idiosyncratic biases. Here we investigated whether individual differences in response bias reflect subject-by-subject idiosyncrasies in sensory encoding. We reanalyzed data from three different tasks ( $\mathrm{N}=443,443$, and 498) from two previous papers (Haddara \& Rahnev, 2020; Rouault et al., 2018). We confirmed the existence of stable individual differences in response bias, and, critically, found that the response bias reflected the idiosyncratic sensory encoding of individual subjects. These results demonstrate that response bias is not simply a "bug" (Summerfield \& $\mathrm{Li}, 2018$ ) but that it follows normative principles that inform how one should respond given how sensory information is represented internally.

\section{The sources of idiosyncratic bias in perceptual decision making}

Several previous studies have investigated idiosyncratic biases and have proposed different sources for them. One line of research has suggested that individual biases may arise from inhomogeneities in how visual information is processed in different neuronal populations in the visual cortex (Afraz et al., 2010). This proposal can explain various idiosyncratic perceptual biases that depend on the spatial location of a stimulus such as biases in gender and age identification (Afraz et al., 2010), in judgments of the direction of optic flow (Wexler et al., 2015), and in object localization (Kosovicheva \& Whitney, 2017). In this view, response bias as an 
inevitable phenomenon arising directly from the limitations in sensory processing, and therefore this proposal is conceptually different from the view that response biases may partly reflect normative principles.

An alternative account of the existence of stable response biases is that they are due to stable dispositions that can be characterized as individual traits (Kantner \& Lindsay, 2012, 2014). This view is based on studies that show that individuals have similar biases in old-new memory tasks across time and even across different tasks. Even though the bias of different subjects in these tasks did not correlate with other stable personality measures, the authors argued that bias in this context is best characterized as a trait-like predisposition.

Here we argue that beyond being an inevitable consequence of inhomogeneous neural processing or an individual predisposition, bias also reflects normative strategies based on the idiosyncrasies of information encoding for each subject. We note that these three explanations of response bias are not mutually exclusive. For example, it is possible that bias related to, for example, choosing red over blue could be simultaneously due to (i) inevitable individual differences in the strength of neural activations induced by each color, (ii) personal preference for choosing one color over the other that are fully independent of the sensory information, and (iii) normative strategies that consider the form of the internal encoding for blue and red colors. Therefore, our findings should not be taken to imply that response bias is optimal or that it is exclusively driven by normative principles. However, our results 
demonstrate that response bias is not simply a failing of the sensory or decisional systems and can, in fact, be the result of an adaptive process that is well adjusted to the individual differences in information processing.

\section{Differences from the ideal observer model}

It should be noted that our analyses implemented the standard assumption that subjects place a single decision criterion directly on the evidence axis. However, this analysis ignores the fact that in cases of unequal variance, the two Gaussian distributions intersect at two different points, and therefore the ideal observer places two separate decision criteria on the evidence axis (Knoblauch \& Maloney, 2012). Nevertheless, for the subjects in our experiments, placing two criteria would only result in a different response once every 1,097 trials, and is thus unlikely to meaningfully alter our results. More importantly, it is questionable whether human subjects can implement the complex decision strategy required by the optimal observer, especially in experiments with confidence ratings (Macmillan \& Creelman, 2005; Rahnev \& Denison, 2018). Therefore, we do not claim that human subjects implement the optimal decision strategy and this question is outside of the scope of this paper. Instead, what our results show is that humans are sensitive to their idiosyncratic sensory encoding even if their response strategy falls short of optimality.

\section{$\underline{\text { Limitations }}$}


Although our results were replicated in three independent tasks, the actual effect sizes observed were modest. Indeed, the correlation between the actual and optimal criterion was only $r=.19$. This modest correlation suggests that while humans do take their idiosyncratic sensory encoding into account when setting their decision criterion, this may only have a small influence on the final decision criterion. Nevertheless, additional simulations suggest that due to small numbers of trials per subject, and the accompanying estimation noise, the maximum correlation one could expect is only about twice as large (Supplementary Figure 2). Therefore, the modest effect size in our data is not a reliable indicator of the importance of the internal sensory distributions in the setting of the response criterion (ideally, what is needed for a reliable estimate is a study with both a very large number of subjects and a very large number of trials per subject). Ultimately, while our results are a strong indicator that subjects are sensitive to the idiosyncratic differences in sensory encoding are taken into account in decision making, it is possible that their influence is relatively small and that the other factors already discussed have a larger influence on the criterion.

Another limitation of our study is that it cannot reveal exactly how subjects learned the relative variability of the internal distributions. Given that the different tasks had between 150 and 330 trials per subject, the learning process likely relied on heuristics that can be applied even after a few trials early in the experiment. Nevertheless, the exact mechanisms of how the criterion is learned over the course of an experiment remain to be described. Finally, while we have used standard 
techniques to estimate the relative variability of the two internal distributions (Macmillan \& Creelman, 2005), future studies may benefit from an independent estimation of the relative variability using a task like magnitude estimation (Petzschner, Glasauer, \& Stephan, 2015).

Conclusion

Using three large datasets (all $\mathrm{N}>400$ ), we found that human perceptual decision making reflects one's idiosyncratic sensory encoding. These results demonstrate that the observation that different subjects have different biases on the exact same task is partly explained by normative considerations. 


\section{Acknowledgements}

This work was supported by the NIH grant R01MH119189. 


\section{References}

Afraz, A., Pashkam, M. V., \& Cavanagh, P. (2010). Spatial heterogeneity in the perception of face and form attributes. Current Biology, 20(23), 2112-2116. https://doi.org/10.1016/j.cub.2010.11.017

Ashby, F. G., \& Maddox, W. T. (2005). Human Category Learning. Annual Review of Psychology, 56(1), 149-178. https://doi.org/10.1146/annurev.psych.56.091103.070217

Fetsch, C. R., Odean, N. N., Jeurissen, D., El-Shamayleh, Y., Horwitz, G. D., \& Shadlen, M. N. (2018). Focal optogenetic suppression in macaque area MT biases direction discrimination and decision confidence, but only transiently. ELife, 7. https://doi.org/10.7554/eLife.36523

Finlayson, N. J., Papageorgiou, A., \& Schwarzkopf, D. S. (2017). A new method for mapping perceptual biases across visual space. Journal of Vision, 17(9), 5. https://doi.org/10.1167/17.9.5.doi

Forstmann, B. U., Ratcliff, R., \& Wagenmakers, E.-J. (2016). Sequential Sampling Models in Cognitive Neuroscience: Advantages, Applications, and Extensions. Annual Review of Psychology, 67, 641-666. https://doi.org/10.1146/annurevpsych-122414-033645

García-Pérez, M. A., \& Alcalá-Quintana, R. (2011). Interval bias in 2AFC detection tasks: sorting out the artifacts. Attention, Perception, \& Psychophysics, 73(7), 2332-2352. https://doi.org/10.3758/s13414-011-0167-x

Green, D. M., \& Swets, J. A. (1966). Signal detection theory and psychophysics. New York: John Wiley \& Sons Ltd. 
Haddara, N., \& Rahnev, D. (2020). The impact of feedback on perceptual decision making and metacognition: Reduction in bias but no change in sensitivity. PsyArXiv. https://doi.org/10.31234/OSF.IO/P8ZYW

Iemi, L., \& Busch, N. A. (2018). Moment-to-Moment Fluctuations in Neuronal Excitability Bias Subjective Perception Rather than Strategic Decision-Making. ENeuro, 5(3), ENEURO.0430-17.2018. https://doi.org/10.1523/ENEURO.043017.2018

Jin, M., \& Glickfeld, L. L. (2019). Contribution of Sensory Encoding to Measured Bias. Journal of Neuroscience, 39(26), 5115-5127. https://doi.org/10.1523/JNEUROSCI.0076-19.2019

Kantner, J., \& Lindsay, D. S. (2012). Response bias in recognition memory as a cognitive trait. Memory \& Cognition, 40(8), 1163-1177. https://doi.org/10.3758/s13421-012-0226-0

Kantner, J., \& Lindsay, D. S. (2014). Cross-situational consistency in recognition memory response bias. Psychonomic Bulletin \& Review, 21(5), 1272-1280. https://doi.org/10.3758/s13423-014-0608-3

Knoblauch, K., \& Maloney, L. T. (2012). Modeling Psychophysical Data in R. https://doi.org/10.1007/978-1-4614-4475-6

Kosovicheva, A., \& Whitney, D. (2017). Stable individual signatures in object localization. Current Biology, 27(14), R700-R701. https://doi.org/10.1016/j.cub.2017.06.001

Linares, D., Aguilar-Lleyda, D., \& López-Moliner, J. (2019). Decoupling sensory from decisional choice biases in perceptual decision making. ELife, 8, e43994. 
https://doi.org/10.7554/eLife.43994

Macmillan, N. A., \& Creelman, C. D. (2005). Detection Theory: A User's Guide (2nd ed.). Mahwah, NJ: Erlbaum.

McKinley, S. C., \& Nosofsky, R. M. (1995). Investigations of exemplar and decision bound models in large, ill-defined category structures. Journal of Experimental Psychology: Human Perception and Performance, 21(1), 128-148. https://doi.org/10.1037/0096-1523.21.1.128

Moutsiana, C., de Haas, B., Papageorgiou, A., van Dijk, J. A., Balraj, A., Greenwood, J. A., \& Schwarzkopf, D. S. (2016). Cortical idiosyncrasies predict the perception of object size. Nature Communications, 7(1), 12110. https://doi.org/10.1038/ncomms12110

Petzschner, F. H., Glasauer, S., \& Stephan, K. E. (2015). A Bayesian perspective on magnitude estimation. Trends in Cognitive Sciences, 19(5), 285-293. https://doi.org/10.1016/j.tics.2015.03.002

Rahnev, D. (2020). Data and codes for paper "Response bias reflects individual differences in sensory encoding." https://doi.org/10.17605/OSF.IO/R4GZ3

Rahnev, D., \& Denison, R. N. (2018). Suboptimality in Perceptual Decision Making. Behavioral and Brain Sciences, 41(e223), 1-66. https://doi.org/10.1017/S0140525X18000936

Rahnev, D., Desender, K., Lee, A. L. F., Adler, W. T., Aguilar-Lleyda, D., Akdoğan, B., ... Zylberberg, A. (2020). The Confidence Database. Nature Human Behaviour, 4(3), 317-325. https://doi.org/10.1038/s41562-019-0813-1

Rahnev, D., Koizumi, A., McCurdy, L. Y., D’Esposito, M., \& Lau, H. (2015). Confidence 
Leak in Perceptual Decision Making. Psychological Science, 26(11), 1664-1680. https://doi.org/10.1177/0956797615595037

Rahnev, D., Maniscalco, B., Graves, T., Huang, E., De Lange, F. P., \& Lau, H. (2011). Attention induces conservative subjective biases in visual perception. Nature Neuroscience, 14(12), 1513-1515. https://doi.org/10.1038/nn.2948

Rahnev, D., Nee, D. E., Riddle, J., Larson, A. S., \& D’Esposito, M. (2016). Causal evidence for frontal cortex organization for perceptual decision making. Proceedings of the National Academy of Sciences, 113(20), 6059-6064. https://doi.org/10.1073/pnas.1522551113

Rouault, M., Seow, T., Gillan, C. M., \& Fleming, S. M. (2018). Psychiatric Symptom Dimensions Are Associated With Dissociable Shifts in Metacognition but Not Task Performance. Biological Psychiatry, 84(6), 443-451. https://doi.org/10.1016/j.biopsych.2017.12.017

Summerfield, C., \& Li, V. (2018). Perceptual suboptimality: Bug or feature? Behavioral and Brain Sciences, 41, e245. https://doi.org/10.1017/S0140525X18001437

Wei, X.-X., \& Stocker, A. A. (2017). Lawful relation between perceptual bias and discriminability. Proceedings of the National Academy of Sciences, 114(38), 10244-10249. https://doi.org/10.1073/pnas.1619153114

Wexler, M., Duyck, M., \& Mamassian, P. (2015). Persistent states in vision break universality and time invariance. Proceedings of the National Academy of Sciences, 112(48), 14990-14995. https://doi.org/10.1073/pnas.1508847112 